\title{
The repopulation of electronic states upon vibrational excitation of niobium carbide clusters
}

\author{
V.Chernyy, ${ }^{1}$ R. Logemann, ${ }^{1}$ J. M. Bakker, ${ }^{2,}$ a) and A. Kirilyuk ${ }^{1,}$ b) \\ 1) Radboud University, Institute for Molecules and Materials, Heyendaalseweg 135, 6525 AJ Nijmegen, Netherlands \\ ${ }^{2)}$ Radboud University, Institute for Molecules and Materials, FELIX Laboratory, Toernooiveld 7c, 6525 ED \\ Nijmegen, Netherlands
}

(Dated: 20 April 2017)

We study the infrared (IR) resonant heating of neutral niobium carbide clusters probed through ultraviolet (UV) photoionization spectroscopy. The IR excitation not only changes the photoionization spectra for the photon energies above the ionization threshold, but also modulates ion yield for energies significantly below it. An attempt to describe the experimental spectra using either Fowler's theory or thermally populated vibrational states was not successful. However, the data can be fully modeled by vibrationally and rotationally broadened discrete electronic levels obtained from Density Functional Theory (DFT) calculations. The application of this method to spectra with different IR pulse energies not only yields information about the excited electronic states in the vicinity of the HOMO level, populated by manipulation of the vibrational coordinates of a cluster, but also can serve as an extra indicator for the cluster isomeric structure and corresponding DFT-calculated electronic levels.

PACS numbers: 63.20.kd,61.46.Bc,63.22.Kn.

\section{INTRODUCTION}

The conventional picture of a solid is that based on the Born-Oppenheimer approximation, which takes into account that typical ionic vibrational frequencies are smaller than about $0.01 E_{F} / \hbar$, with $E_{F}$ the Fermi energy ${ }^{1}$. In other words, electron velocities are orders of magnitude larger than those of ions. As a result, the electrons adiabatically adjust to the moving ions at each moment of time. In this picture, the interactions of lattice excitations (phonons) with electrons are described as a small perturbation of the electronic wavefunction by the static, distorted lattice. Although the effects of the electron scattering by these perturbations are small, they result in dramatic phenomena such as superconductivity, polarons, etc.

In many cases, however, non-adiabatic processes may play a significant role. Such are, for example, conical intersections of energy surfaces in molecules, ${ }^{2}$ superconducting cuprates, ${ }^{3}$ or graphene. ${ }^{4}$ In these cases, the energies of electronic and vibrational excitations become comparable, leading to strongly amplified interactions and a considerable renormalization of energies. Additionally, the direct energy transfer between the systems becomes possible.

Atomic clusters bridge the gap between atomic/molecular and bulk states of matter, with as unique characteristic that the physico-chemical properties strongly vary on an atom-by-atom level ${ }^{5,6}$. Clusters contain all the components that solids are made of, but their properties are controllable and a complete theoretical description can be developed. Unlike in

\footnotetext{
a)Electronic mail: j.bakker@science.ru.nl

b)Electronic mail: a.kirilyuk@science.ru.nl
}

solids, the electronic and vibrational states in clusters are discrete, as no bands can be formed. Moreover, while in solids the energy transitions of electrons can appear separately from the excitation of the other subsystems, in clusters and molecules, in accordance to the Franck-Condon principle, the change of the electronic quantum state is not possible without incorporation of the overlap between vibrational wave functions.

"Bulk-like" effects such as non-adiabatic energy transfer between the electronic and vibrational systems in clusters have been studied for decades ${ }^{7-13}$. Usually a direct excitation of the cluster electronic system is performed with subsequent observation of the relaxation time through the energy exchange with the nuclear system. The reverse experiment, where the cluster nuclear coordinates are excited in the electronic ground state and the energy is transferred to electronically excited states, which can eventually lead to ionization, was primarily used to study the vibrational properties of clusters ${ }^{14,15}$. Recently, we presented a study demonstrating the possibility to probe the population redistribution over lowlying electronic levels using UV photoionization ${ }^{16}$.

In this work we study the non-adiabatic energy transfer from excited vibrational states into the electronic system in gas-phase niobium carbide clusters. Bulk NbC is a superconductor, which is an indication of a strong electron-phonon interaction. Moreover, it was previously demonstrated that $\mathrm{NbC}$ clusters, upon resonant pumping of vibrational coordinates, exhibit thermionic emission, which is a direct proof of the presence of the coupling between vibrational and electronic coordinates. ${ }^{17}$

Experimentally, vibrational energy is transferred to the electronic system after a controlled excitation of the vibrational coordinates. The energy transfer results in a change of the population of the electronic density of states in niobium carbide clusters, which is reflected by 
a modification of the photoionization spectra. To semiquantitatively explain the observed results, we construct a simple model where the electronic population after excitation is thermally re-distributed over the electronic levels close to the HOMO level. The electronic levels are obtained from DFT calculations. To ensure the electronic levels are calculated for the proper cluster geometry, we require knowledge about their structures. To this end, we first record IR spectra for the NbC clusters using IR-UV two-color spectroscopy ${ }^{18}$. We determine the geometric structure by evaluating the correspondence between the experimental IR spectra and spectra for trial geometries calculated using DFT.

The paper is organized as follows: after presenting the used experimental and computational techniques, we first discuss IR spectra of niobium carbide clusters at a fixed UV photoionization wavelength and determine their geometric structures. We then turn to the influence of IR excitation on the photoionization spectra. The observed photoionization spectra are subsequently interpreted using several models, and we demonstrate that the model employing electronic states extracted from the DFT calculations gives the most satisfactory result. We conclude by a discussion on the observed temperatures and the opportunities these results will give for experimental studies on the dynamics of electron-phonon coupling in clusters.

\section{EXPERIMENTAL AND COMPUTATIONAL DETAILS}

The experiments are carried out in an instrument coupled to the Free Electron Laser for Infra-red eXperiments $(\text { FELIX })^{19}$. Clusters are formed by ablation of a rotating niobium rod (Sigma Aldrich) using a pulsed $532 \mathrm{~nm}$ $\mathrm{Nd}^{2+}$ :YAG laser with an energy of $\sim 10 \mathrm{~mJ}$ per pulse in the presence of the gas mixture (6 bar) of $\mathrm{He}(99 \%)$ and methane (1\%) injected by a pulsed valve (General Valve, Series 9). The formation takes place at room temperature within a growth channel ( $4 \mathrm{~mm}$ diameter) and after passing an extension tube, the mixture expands into vacuum. The molecular beam is skimmed upon entering a differentially pumped chamber where charged species are deflected out by a pair of electrically biased metal plates. The beam of neutral clusters is further shaped by a $1 \mathrm{~mm}$ diameter aperture and enters an interaction chamber with the extraction source of a reflectron timeof-flight mass spectrometer (R.M. Jordan TOF products, inc.).

Approximately $25 \mathrm{~mm}$ before the center of the extraction region, clusters interact with the loosely focused IR light from FELIX that counter propagates the cluster beam. At this point the laser waist is comparable to the size of the molecular beam, ensuring that the majority of the clusters interact with the IR beam.

FELIX produces IR radiation in the range of $66-3600$ $\mathrm{cm}^{-1}$. The laser light is emitted in a $\sim 9 \mu$ s pulse train (called macropulse) of picosecond duration pulses. The spectral bandwidth is transform-limited and is ad- justable between $0.2-1 \%$ RMS of the central frequency. The typical macropulse energies produced by FELIX are up to $100 \mathrm{~mJ}$. The pulse energy can be varied over four orders of magnitude with fixed-value attenuators. After approximately $35 \mu \mathrm{s}$, the clusters are in the center of the extraction region where they are irradiated by a frequency-doubled dye laser (Lioptec, 269-236 nm). All ions formed are accelerated into the flight region of the mass-spectrometer and detected by a microchannel detector.

Two types of experiments are presented. First, IR spectra are recorded by varying the FELIX frequency while keeping the UV photoionization wavelength constant. Second, the influence of the IR heating is monitored by keeping the IR frequency fixed and varying the photoionization frequency. To monitor the influence of the IR laser, the experiment operates at twice the FELIX frequency, thus allowing for the alternating recording of two mass spectra for each UV wavelength: one with and one without IR excitation. To ensure that the UV photoionization spectrum is not contaminated by contributions due to multiphoton ionization, the UV laser pulse energy was attenuated to $15 \mu \mathrm{J}$. The UV laser pulse energy for IR spectra was fixed at $150 \mu \mathrm{J}$, giving a useful amount of multiphoton ions (see below).

To interpret the experimental results, DFT calculations are carried out using the Vienna ab-initio simulation package (VASP) ${ }^{20}$ using the projector augmented wave (PAW) method ${ }^{21,22}$ and the Perdew, Burke and Ernzerhof (PBE) functional ${ }^{23}$. The search for the lowest energy structures was done by a genetic algorithm ${ }^{24}$ (GA) in combination with DFT. This method has already demonstrated excellent results in case of $\mathrm{Fe}_{\mathrm{x}} \mathrm{O}_{\mathrm{y}}{ }^{+}$ clusters $^{25}$.

Standard recommended PAWs with an energy cutoff of $400.0 \mathrm{eV}$ are used. All forces were minimized below $10^{-3} \mathrm{eV} / \AA$. The clusters are placed in a periodic box of a size of 12 and $14 \AA$ for $\mathrm{Nb}_{3} \mathrm{C}_{2}$ and $\mathrm{Nb}_{6} \mathrm{C}_{5}$ respectively, which we checked to be sufficiently large to eliminate intercluster interactions for each cluster size. For the calculations a single $\mathbf{k}$ point $(\Gamma)$ is used. In addition, for $\mathrm{Nb}_{6} \mathrm{C}_{5}$ all possible combinations of carbon positions between the $\mathrm{Nb}$ atoms were geometrically optimized to test the genetic algorithm results. For the three isomers lowest in energy the structure was reoptimized using higher accuracy. Subsequently, the electronic density of states and vibrational frequencies were calculated. All frequencies presented are unscaled. Zero-point vibrational energies (ZPVE) were calculated for the three lowest in energy isomers found for each cluster size.

\section{RESULTS}

\section{A. IR spectra}

We present IR spectra for $\mathrm{Nb}_{3} \mathrm{C}_{2}$ and $\mathrm{Nb}_{6} \mathrm{C}_{5}$. Both spectra are recorded with the UV photoionization laser 


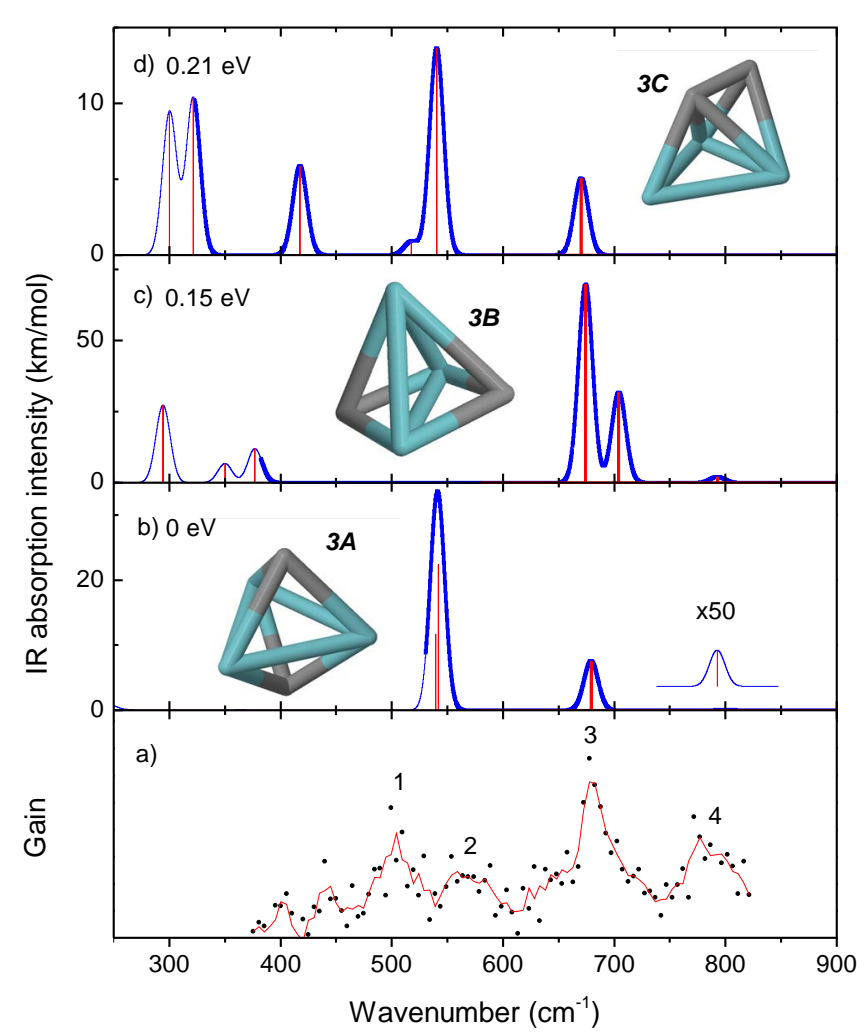

FIG. 1. Experimental (panel (a), circles) and calculated (b-d) IR spectra of $\mathrm{Nb}_{3} \mathrm{C}_{2}$ clusters. The red line is three-point adjacent average of the experimental data. Calculated discrete vibrational frequencies (red vertical lines) are convoluted with a $15 \mathrm{~cm}^{-1}$ FWHM Gaussian line shape function (blue). Color coding corresponding geometries: C-gray, and Nb-green. The energy differences include ZPVE.

fixed at $250.5 \mathrm{~nm}$, or $4.95 \mathrm{eV} /$ photon, which is close to the value for the previously reported Ionization Energy (IE) for $\mathrm{Nb}_{3} \mathrm{C}_{2}$ of 4.96(0.06) and a bit lower than the IE of 5.1(0.1) eV reported for $\mathrm{Nb}_{6} \mathrm{C}_{5}{ }^{26}$. The spectral region of interest in this work is $400-800 \mathrm{~cm}^{-1}$, where the majority of the vibrational modes predicted by DFT calculations is found. The spectra are recorded with IR pulse energies in the range of 8 to $11 \mathrm{~mJ}$.

Since the production of clusters can exhibit fluctuations, and we aim to maximize visibility of subtle changes in the cluster ion yield in the presence of an (often larger) background signal, it is desirable to choose the ratio of the number of detected ions with both IR and UV lasers to that detected with UV laser only. The situation with no response thus yields a ratio of 0 . Hence, the spectra obtained are presented as the experimental gain function defined as:

$$
G_{\mathrm{EXP}}^{I R}(\omega)=\frac{I_{\mathrm{IR}+\mathrm{UV}}(\omega)-I_{\mathrm{UV}}(\omega)}{I_{\mathrm{UV}}(\omega)},
$$

where $I_{\mathrm{IR}+\mathrm{UV}}(\omega)$ and $I_{\mathrm{UV}}(\omega)$ are the number of ions produced with and without IR radiation at frequency $\omega$, respectively. Here, $\mathrm{I}_{U V}(\omega)$ is depicted as a function of $\omega$ to indicate that this yield is recorded concurrent with $\mathrm{I}_{I R+U V}(\omega)$ and can account for long-term source fluctuations. Note, that for the UV pulse energy of $150 \mu \mathrm{J}$ that we use to obtain IR spectra $I_{\mathrm{UV}}(\omega)>0$, due to the amount of signal from the multiphoton ionization, which allows to avoid divergence of the $G_{\mathrm{EXP}}^{I R}(\omega)$. The experimental IR spectra are further normalized on the IR laser pulse energy.

$\mathrm{Nb}_{\mathbf{3}} \mathbf{C}_{\mathbf{2}}$. The experimental vibrational spectrum for $\mathrm{Nb}_{3} \mathrm{C}_{2}$ is depicted in Fig. 1(a). The spectrum is not very well resolved, but we can clearly discriminate at least four clear resonances, which are indicated in Fig. 1(a) at 505, 565,670 and $780 \mathrm{~cm}^{-1}$. The strongest band is the one at $680 \mathrm{~cm}^{-1}$, which is in agreement with the pattern observed for other NbC clusters studied using resonant IR ionization spectroscopy ${ }^{27}$. Upon further comparison with spectra obtained using the latter technique, we note that the current spectrum is much better resolved, which is due to the more sensitive nature of IR-UV spectroscopy, requiring substantially lower IR laser fluences.

In panels (b-d) of Fig. 1 the three lowest energy isomers found using the GA are shown. Two of these (structures $3 \mathrm{~A}$ and $3 \mathrm{~B}$ ), were proposed previously ${ }^{28}$. Structure $3 \mathrm{~A}$ is the lowest in energy and has a slightly distorted trigonal bipyramid shape and is formed by a triangular $\mathrm{Nb}$ basis capped by two $\mathrm{C}$ atoms. The $\mathrm{Nb}-\mathrm{Nb}$ distances are 2.46, 2.49 and $2.59 \AA$, and the $\mathrm{Nb}-\mathrm{C}$ bond lengths are $2.03,2.04$ and $2.05 \AA$, respectively. Structure $3 \mathrm{~B}$ is 0.15 $\mathrm{eV}$ higher in energy than $3 \mathrm{~A}$. Having a $C_{2 v}$ point group symmetry, this isomer has a squared pyramidoid shape with a $\mathrm{Nb}_{2} \mathrm{C}_{2}$ rhombic base. $\mathrm{Nb}-\mathrm{Nb}$ distances in this isomer are 2.41 and $2.45 \AA$, for the base and between base and top, respectively, and the $\mathrm{Nb}-\mathrm{C}$ distances are 2.33 and $1.96 \AA$.

Isomer $3 \mathrm{C}$ is $0.21 \mathrm{eV}$ higher in energy than $3 \mathrm{~A}$ and has a $C_{s}$ point symmetry group. It consists of a trigonal pyramid, with a $\mathrm{Nb}_{3}$ triangular base and a $\mathrm{C}$ top. The second $\mathrm{C}$ is capping one of the planes of the pyramid. The $\mathrm{Nb}-\mathrm{Nb}$ bond lengths are 2.45 and $2.43 \AA$, the $\mathrm{C}-\mathrm{C}$ bond is $1.37 \AA$, and the $\mathrm{Nb}-\mathrm{C}$ bonds vary from 2.0 to 2.19 Å.

From Fig. 1 it can be clearly seen that every calculated spectrum has common vibrational frequencies with the experimental one. All spectra exhibit one or multiple bands close to $670 \mathrm{~cm}^{-1}$, but none of them matches perfectly with the experimental data. We nevertheless assign our spectrum to structure $3 \mathrm{~A}$, for the following reasons: first, its calculated spectrum predicts vibrational modes close to each experimentally observed band, whereas structure $3 \mathrm{~B}$ has no bands in the $400-650 \mathrm{~cm}^{-1}$ range, and structure $3 \mathrm{C}$ lacks a band between $700-900 \mathrm{~cm}^{-1}$. It is clear that the intensities predicted do not match with the experiment very well. Part of the mismatch could lie in the much larger experimental frequency difference of $60 \mathrm{~cm}^{-1}$ between bands 1 and 2, which are near-degenerate for the calculated spectrum $\left(2 \mathrm{~cm}^{-1}\right)$. One would be tempted to argue that structures $3 \mathrm{~B}$ and $3 \mathrm{C}$ also contribute to the experimental vibrational spec- 


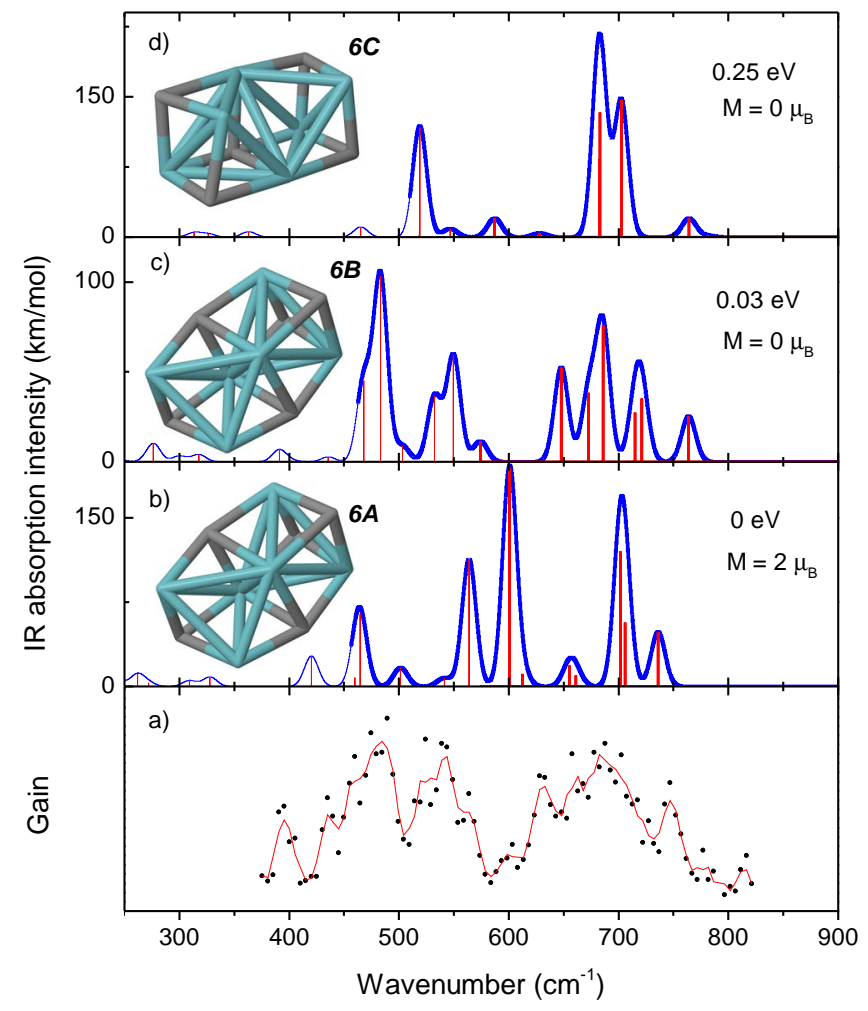

FIG. 2. Experimental (panel (a), circles) and calculated (b-d) IR spectra of $\mathrm{Nb}_{6} \mathrm{C}_{5}$ clusters. The red line is three-point adjacent average of the experimental data. Calculated discrete vibrational frequencies (red vertical lines) are convoluted with a $15 \mathrm{~cm}^{-1}$ FWHM Gaussian line shape function (blue). Color coding corresponding geometries: C-gray, and Nb-green. The energy differences include ZPVE.

trum. However, we will demonstrate below that using the UV photoionization dependence it is possible to get an additional confirmation for assigning the spectrum for $\mathrm{Nb}_{3} \mathrm{C}_{2}$ to structure $3 \mathrm{~A}$ only. This conclusion is consistent with previous investigations ${ }^{28}$.

$\mathbf{N b}_{\mathbf{6}} \mathbf{C}_{\mathbf{5}}$. Fig. 2(a) shows the experimental vibrational spectrum for $\mathrm{Nb}_{6} \mathrm{C}_{5}$. Two broad but structured bands can be seen in the spectrum in the ranges 350-570 and $570-750 \mathrm{~cm}^{-1}$, respectively. In the $350-570 \mathrm{~cm}^{-1}$ range, we distinguish at least three peaks that are partly resolved. Their maxima are at 395, 467, and $523 \mathrm{~cm}^{-1}$. The second band contains peaks that are less well resolved, but we identify their maxima at 632, 686, and $747 \mathrm{~cm}^{-1}$, with the middle band the main carrier of intensity.

The calculated vibrational spectra for several isomers are shown in Fig. 2(b-d). All structures consist of two $\mathrm{Nb}$ tetrahedra that share one edge. In structures $6 \mathrm{~A}$ and $6 \mathrm{~B}$ two sets of $\mathrm{Nb}$ atoms not on the shared edge are connected through a carbon bridge, while the three remaining carbons cap the tetrahedra planes, of which one thus remains uncapped: the one facing the reader. One can also imagine the geometry as constructed out of two distorted cubes, containing both $\mathrm{Nb}$ and $\mathrm{C}$, one of which has one carbon missing in the vertex. Interestingly, the two lowest in energy candidates, structure $6 \mathrm{~A}$ and $6 \mathrm{~B}$, have the same geometric configuration but differ in spin configuration, which leads to small differences in bond lengths.

Structure $6 \mathrm{~A}\left(2 \mu_{B}\right)$ is the lowest in energy and has $\mathrm{Nb}-\mathrm{Nb}$ bond lengths ranging from 2.45 to $2.90 \AA$ and for $\mathrm{Nb}-\mathrm{C}$ from 1.98 to $2.17 \AA$. Structure $6 \mathrm{~B}\left(0 \mu_{B}\right)$, only $0.03 \mathrm{eV}$ higher in energy, has $\mathrm{Nb}-\mathrm{Nb}$ bond lengths varying from 2.51 to $2.91 \AA$ and $\mathrm{Nb}-\mathrm{C}$ bond lengths varying from 1.94 to $2.24 \AA$. Structure $6 \mathrm{C}$ at $0.25 \mathrm{eV}$ is quite similar to $6 \mathrm{~A}$ and $6 \mathrm{~B}$ but lacks one of the carbon bridges, and each outward facing tetrahedron face is now $\mathrm{C}$-capped. $\mathrm{Nb}-\mathrm{Nb}$ bond lengths for this isomer vary from 2.68 to $2.92 \AA$, and for Nb-C from 1.94 to $2.18 \AA$.

Fig. 2 allows us to compare the calculated spectra for different isomers with the experimental result. It appears that some of the strongest resonances on the experimental spectrum are saturated. Nevertheless, we are able to distinguish all the peaks predicted by theory in the experimental spectrum: there is an excellent agreement for structure $6 \mathrm{~B}$, both in frequencies and intensities of the predicted vibrations. Structure $6 \mathrm{~A}$ is ruled out by the strong band at $600 \mathrm{~cm}^{-1}$; structure $6 \mathrm{C}$ has better similarities to the experimental spectrum, but the match is not as good as for $6 \mathrm{~B}$. This allows us to conclude that there is a good correspondence between theory and experiment and we assign the experimental spectrum to structure $6 \mathrm{~B}$ with zero magnetic moment.

\section{B. IR-induced modification of UV photoionization spectra}

After determination of the vibrational bands, the IR laser was set to $\omega=675 \mathrm{~cm}^{-1}$ (a common resonance for the majority of the investigated species including $\mathrm{Nb}_{3} \mathrm{C}_{2}$ and $\mathrm{Nb}_{6} \mathrm{C}_{5}$ ) and photoionization spectra were recorded. Note that we use $\nu$ for UV frequency to discern it from $\omega$ used for IR frequency. To avoid multiphoton effects, we use a relatively low UV pulse energy $(15 \mu \mathrm{J})$. Under this condition $\mathrm{I}_{U V}(\nu)$ can approach zero in the spectral region well below the ionization threshold and the function $G_{\mathrm{EXP}}^{I R}$ would diverge. For this reason, we present UV gain curves

$$
G_{\mathrm{EXP}}^{U V}(\nu)=\frac{I_{\mathrm{IR}+\mathrm{UV}}(\nu)-I_{\mathrm{UV}}(\nu)}{I_{\mathrm{UV}}(\nu)+I_{0}} .
$$

Here we add a term $I_{0}$ to the denominator, taken as the mean value of the five shortest wavelength data points where the detected signal has reached a near-constant value for the considered spectrum. This protects $G_{\mathrm{EXP}}^{U V}(\nu)$ from divergence when $I_{\mathrm{UV}}(\nu) \approx 0$.

Fig. 3 shows the spectra for $\mathrm{Nb}_{3} \mathrm{C}_{2}$ and $\mathrm{Nb}_{6} \mathrm{C}_{5}$ close to their IEs, over a rather broad energy range. The photoionizaton spectra without IR excitation are shown in black, those with IR excitation in red. IR laser pulse energies are shown in increasing order, as indicated in 


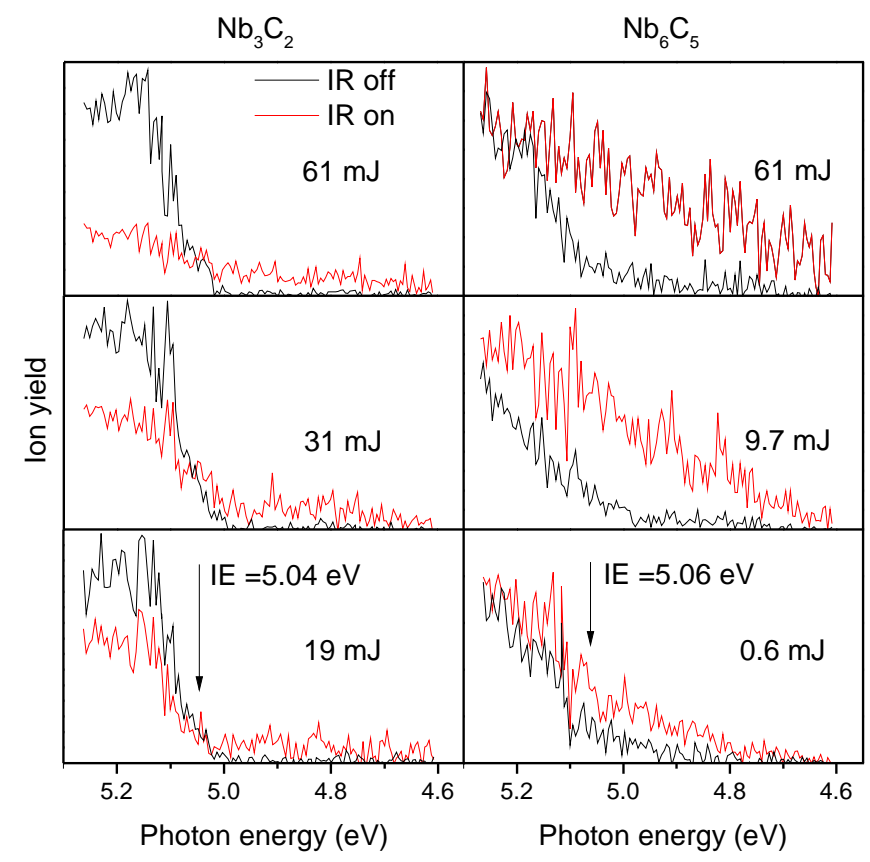

FIG. 3. UV photoionization spectra for $\mathrm{Nb}_{3} \mathrm{C}_{2}$ and $\mathrm{Nb}_{6} \mathrm{C}_{5}$ clusters without (black) and with (red) prior irradiation by FELIX at different IR pulse energies.

the graph. Note that the energy axis is inverted, for reasons that will become clear later. The photionization curves without prior IR excitation show a rather shallow threshold behavior, where the ion yield grows from zero to a maximum over an energy range of approx. $0.2 \mathrm{eV}$ for $\mathrm{Nb}_{3} \mathrm{C}_{2}$ and even $0.4 \mathrm{eV}$ for $\mathrm{Nb}_{6} \mathrm{C}_{5}$. These observations are consistent with previously published photionization spectra for niobium carbide clusters ${ }^{26}$, and for clusters in general, see e.g. Ref. 29. The shallowness of the spectra has been long debated and is commonly attributed to substantial changes in the geometry upon ionization, but also to the thermal population of rovibrational states.

The photoionzation spectra with prior IR excitation exhibit not only a change in the steepness of the slope but also the appearance of the signal at photon energies substantially lower than the IE. For both cluster sizes it can be clearly observed that with increasing IR pulse energy the photoionization curve gets more and more spread to the low-energy side, and some sort of plateau gets formed. The curve for $\mathrm{Nb}_{3} \mathrm{C}_{2}$ shows a substantial decrease of the overall ion yield at higher IR energies, which we attribute to loss due to dissociation and/or direct ionization.

An example for the gain spectrum for $\mathrm{Nb}_{3} \mathrm{C}_{2}$ is shown in the top panel of Fig. 4, recorded with an IR pulse energy of $31 \mathrm{~mJ}$. The figure also shows three different models with which we attempt to semi-quantitatively explain our results. Each model UV gain curve has been generated in two steps: first we construct $I_{U V}$ by fitting the UV curve without IR excitation to the chosen model. In the second step, we construct a model gain function with $I_{U V}$ fixed using the parameters found in step 1. We then

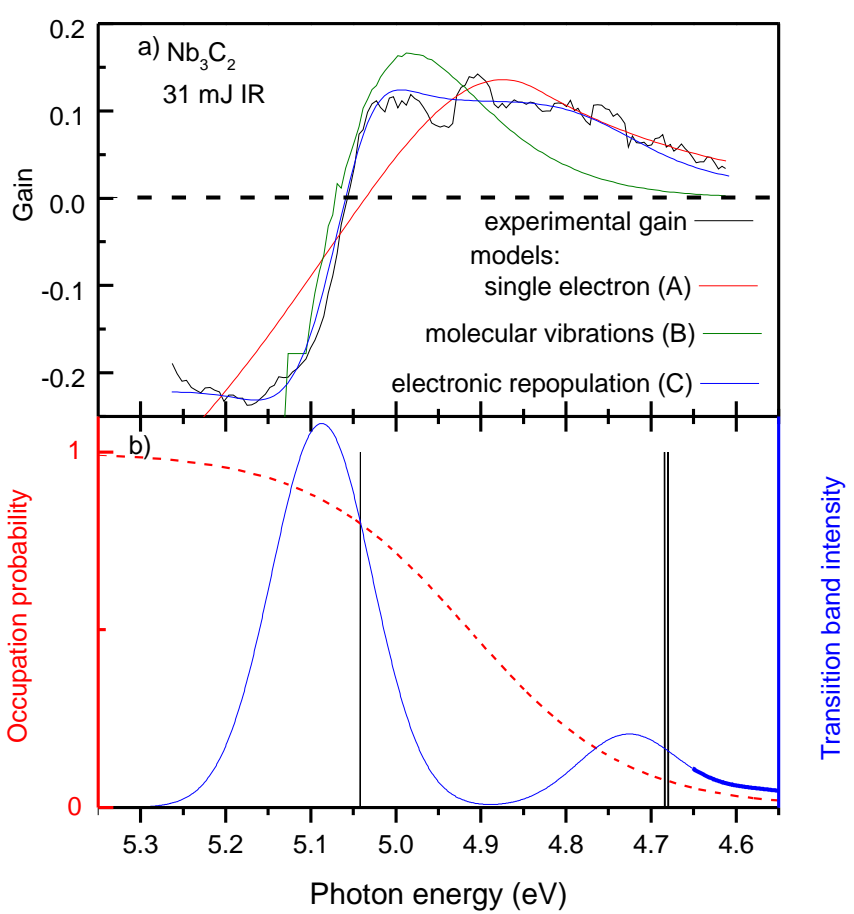

FIG. 4. a) Experimental IR induced gain (black) for $\mathrm{Nb}_{3} \mathrm{C}_{2}$ and model fit curves. b) Calculated electronic density of states for $\mathrm{Nb}_{3} \mathrm{C}_{2}$ (structure $3 \mathrm{~A}$, sticks), the Fermi-Dirac distribution function (1087 K, red dashed line), and fitted transition band structure from approach (C) (blue).

minimize the difference between the experimental and the fit gains varying only the parameters for $I_{U V+I R}$, including a scale factor for $I_{U V+I R}$ to accommodate for the dissociation or direct ionization at the highest UV energies. Note, that constructing the gains we are always consistent: for each model gain we use exactly the same $I_{0}$ as for the corresponding experimental gain. It is then clear that even if the gain function $G_{\mathrm{EXP}}^{U V}$ is sensitive to $I_{0}$, leading to a slight change in the visible representation of the curve, it will be taken into account in the further analysis and will not influence the result of the fit.

We will now discuss each of the models used.

A. Single-electron picture for solids. Fowler's theory ${ }^{30}$ was developed for an infinite metallic 2D surface and is based on the uniform and continuous distribution of the electronic density of states. It has recently been successfully used to describe the photoionization thresholds for some relatively large clusters ${ }^{31}$. The possibility to apply this model to small clusters, where the density of electronic states is rather discrete, seems unlikely. We nevertheless fit a model gain function based on Fowler's approach in order to demonstrate the importance of the discrete levels for the shape of the photoionization curves for small clusters.

Upon absorption of a photon with energy $h \nu$, the flux of electrons $I(E, T)$ leaving the surface of a metal at temperature $T$ is described by: 


$$
I(E, T) \propto T^{2} \cdot f\left(\frac{E}{k_{B} T}\right),
$$

where $E=I E-h \nu$ is the detuning from the ionization energy $I E, k_{B}$ Boltzmann's constant, and $f(x)$ the integral over the Fermi-Dirac distribution function given by a series expansion ${ }^{30}$ :

$$
f(x)= \begin{cases}e^{x}-\frac{e^{2 x}}{4}+\frac{e^{3 x}}{9}-\ldots & x \geq 0 \\ \frac{\pi^{2}}{6}+\frac{x^{2}}{2}-\left(e^{-x}-\frac{e^{-2 x}}{4}+\frac{e^{-3 x}}{9}-\ldots\right) & x \leq 0 .\end{cases}
$$

The result of the fit, where we use $T$ as a fit parameter and the $I E$ is fixed at the value extracted from the fit of the photoionization curve without IR excitation, is depicted by the red line in Fig. 4(a). The correspondence between this theory and experiment is acceptable in the low-energy region (far from the ionization threshold) only; the model is not able to describe the high photon energy side of the spectrum

B. Molecular picture. In Ref. ${ }^{32}$ it was demonstrated that a resonant IR excitation of para amino benzoic acid can lead to an enhanced UV photoionization yield at frequencies below the ionization threshold. As there are no possible electronically excited states within a few $\mathrm{eV}$ of the ground state, the excitation must be purely vibrational, and the enhancement due to an efficiency increase of the ionization probability, presumably simply by the increase of possible ionization pathways. We test whether the same could be true for $\mathrm{Nb}_{3} \mathrm{C}_{2}$ by modeling the gain as the ratio of the number of accessible ionization channels from the thermally populated vibrational densities of states (vDOS) 32,33 , considering all transitions from the populated vDOS to the continuum. In this treatment we do not take into account (unknown) differences in the Franck-Condon factors and assume that the transition probabilities from all the vibrational levels to the continuum are equal. This means that the ion signal at each frequency is only determined by the population of the corresponding energy level.

The vDOS $\rho_{v i b}(\mathrm{E})$ is numerically evaluated using the Beyer-Swinehart algorithm ${ }^{34}$ with DFT-calculated harmonic frequencies as input. The UV photoionization curve for values of $E_{v i b}=I E-h \nu$ higher than zero was constructed as an integral over all populated vibrational states as:

$$
I\left(E_{v i b}, T\right) \propto \int_{E_{v i b}}^{\infty} \rho_{v i b}\left(E_{v i b}\right) \cdot e^{-\frac{E_{v i b}}{k_{B} T}},
$$

and assumed constant for $E_{v i b} \leq 0$ or frequencies above the ionization threshold. In analogy with the previous model, we use $T$ as a fit parameter and $I E$ is fixed at the value extracted from the fit of the photoionization curve without IR excitation. This model is shown in green in Fig. 4(a) and provides a better explanation for the sharp rise in the experimental gain, but it underestimates the experimentally observed behavior for low UV energies. Note that the rising edge of the model shows a stepwise behavior, reflecting the discrete nature of the vDOS at low energies.

C.DFT-based electronic repopulation. In our final model we use the information about the Kohn-Sham orbitals for a cluster obtained from the DFT calculations in combination with the Fermi-Dirac distribution to determine the population of the electronic density of states, as successfully applied for metallic clusters ${ }^{16}$. Inspired by a model of two displaced harmonic oscillators ${ }^{35,36}$, we further assume that transitions from each populated electronic level $E_{i}$ can be simulated by a Gaussian function dubbed transition band. The maximum of the transition band, i.e. the highest probability of the transition between the initial and final states, is blue shifted by a factor $\delta$ with respect to the electronic level, as illustrated in Fig. 4(b).

We match the position of the HOMO level to the Adiabatic IE, determined from the experimental data as the point where the extrapolated first linear rise in the photoionization efficiency spectrum intersects with the baseline $^{6}$. The values obtained this way show a reasonable agreement with those obtained from DFT calculations (as the energy difference between the ground neutral and cationic states) for $\mathrm{Nb}_{3} \mathrm{C}_{2}$ (5.04 experiment and 5.09 DFT, respectively); for $\mathrm{Nb}_{6} \mathrm{C}_{5}$ the experimental value of $5.06 \mathrm{eV}$ deviates from the calculated value 4.63 $\mathrm{eV}$. However, such deviations are not uncommon ${ }^{37}$.

The ion yield is modeled by numerical integration of the transition bands for each populated electronic level:

$$
I(E, T) \propto \sum_{i=1} F\left(E_{i}, T\right) \cdot \operatorname{erf}\left(\sigma\left(E-E_{i}-\delta\right)\right),
$$

with

$$
\operatorname{erf}(x)=\frac{1}{\pi} \int_{-\infty}^{x} e^{-t^{2}} \mathrm{~d} t
$$

where $\sigma$ is the Gaussian width of each transition band, $F(E, T)$ the Fermi-Dirac distribution function and $T$ the electronic temperature. Through the DFT-calculated vibrational frequencies $\omega_{i}, \sigma$ can be expressed $\operatorname{as}^{35}$ :

$$
\sigma^{2}=\sum_{i=1}^{M}\left(\hbar \omega_{i}\right)^{2} \cdot \frac{a^{2}}{2} \cdot \operatorname{coth}\left(\frac{\hbar \omega_{i}}{2 k T}\right),
$$

where $M$ is the number of the vibrational degrees of freedom of a cluster, and $a$ is a coupling parameter characterizing the displacement between the harmonic oscillators for the ionic and ground states ${ }^{35}$, taken to be the same for each vibrational mode of the cluster. This appears to be a reasonable approximation, as the width $\sigma$ of the transition band with fixed $a$ now only depends on $T$. It allows us to incorporate for the effects of population of the vibrational states as well. Our assumption of a fixed displacement $\delta$ for each transition band is strictly speaking also not true due to the difference in Franck-Condon 


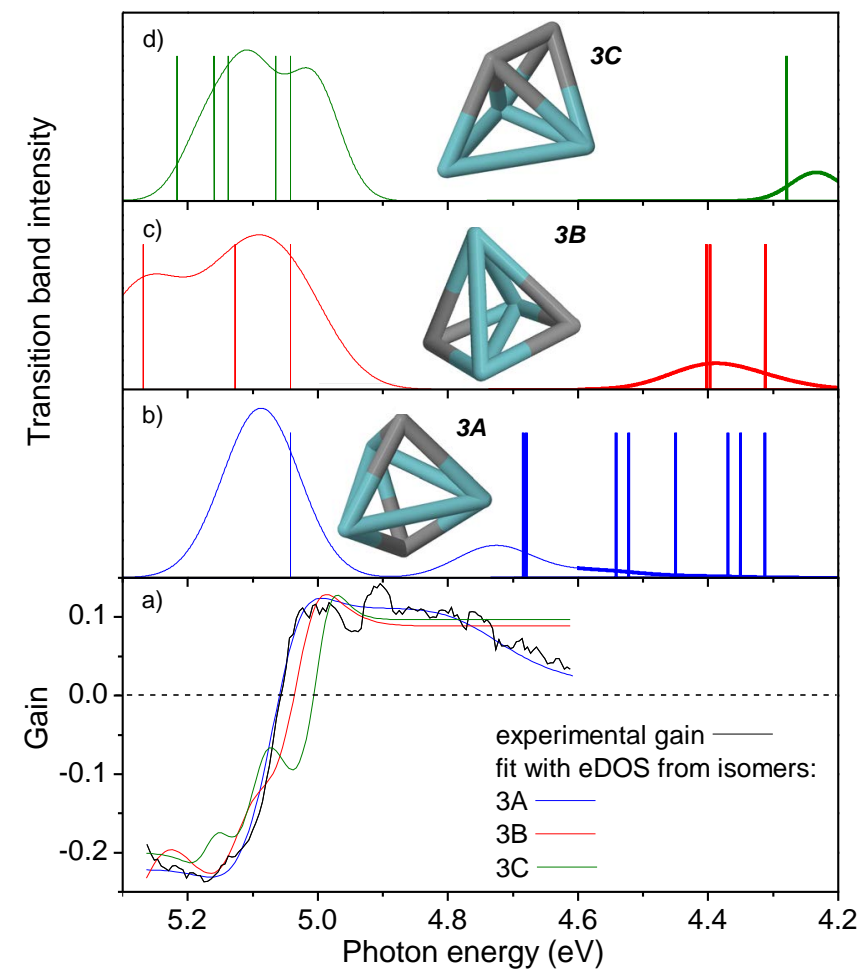

FIG. 5. Figure was modified. Experimental IR induced gain function for $\mathrm{Nb}_{3} \mathrm{C}_{2}$ (panel a, black) and the gain function calculated using the DFT based electron correlation model for structures 3A, 3B and 3C (blue, red and green respectively). The associated transition bands (lines) and DFT calculated energy levels (sticks) for each structure are shown in panels (b-d).

overlaps between vibrational wavefunctions of different electronic states with the ionized state. Nevertheless, these approximations allow us to reduce the number of fit variables from three to one. $a$ and $\delta$ are extracted from the fit of the photoionization curve without IR excitation and are fixed for fitting of the gain curve. Note that the position of the Fermi level in the Fermi-Dirac distribution changes as a function of temperature in order conserve the number of electrons. The resulting gain curve is depicted in Fig. 4(a) by a blue solid line. It successfully describes all features of the gain spectrum.

Model (C) can also be used as a second experimental test for the calculated cluster structure. Fig. 5(a) shows the fit curves using the DFT calculated electronic Density of States (eDOS) for structures 3A, 3B and 3C (blue, red and green respectively). Due to the difference in energies of the electronic levels for the different isomers and in particular the values of the HOMO-LUMO gaps shown in Fig. 5(b-d), the positions of the transition bands will be different for different geometries. In case of $\mathrm{Nb}_{3} \mathrm{C}_{2}$ this leads to an overestimation of the gain in the low energy region (below $4.85 \mathrm{eV}$ ) by the fit curves for both struc-

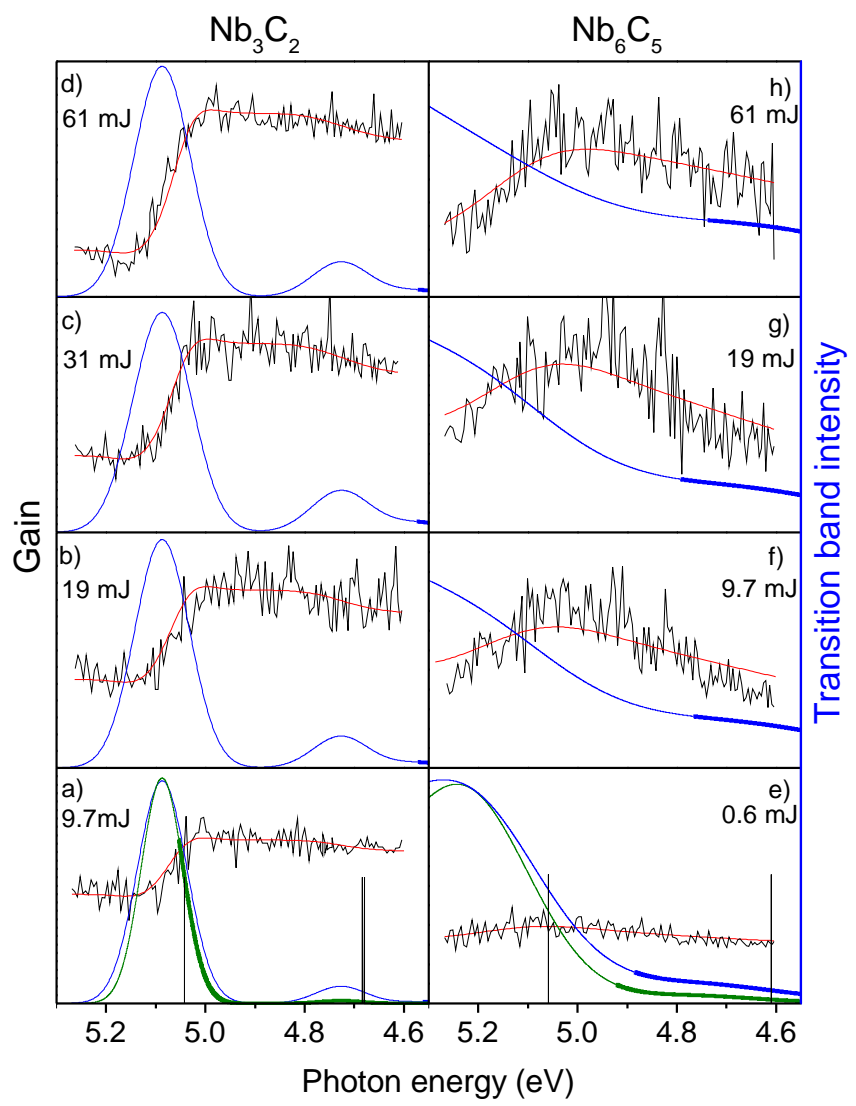

FIG. 6. Experimental (black) and modeled (red) IR induced gain functions for $\mathrm{Nb}_{3} \mathrm{C}_{2}$ and $\mathrm{Nb}_{6} \mathrm{C}_{5}$ for different IR pulse energies. The DFT calculated energy levels are depicted by sticks and the transition bands are shown in blue (excited) and green (unexcited).

tures 3B and 3C. Even though this method cannot be considered as decisive, we consider the result as an extra indication apart from the IR spectra that only isomer $3 \mathrm{~A}$ is observed under the current experimental conditions.

Experimental gain spectra recorded for different IR pulse energies for $\mathrm{Nb}_{3} \mathrm{C}_{2}$ and $\mathrm{Nb}_{6} \mathrm{C}_{5}$ are shown in black in Fig. 6. The fit to the experimental gain using model (C) is shown in red and the corresponding transition band structures for the excited clusters are depicted in blue. For completeness, the band structures for the unexcited cluster are shown in Fig. 6(a) and 6(e) in green, together with the positions of the calculated electronic levels for both clusters (sticks). The calculated values for the HOMO-LUMO energy gaps for $\mathrm{Nb}_{3} \mathrm{C}_{2}$ and $\mathrm{Nb}_{6} \mathrm{C}_{5}$ clusters are 0.36 and $0.45 \mathrm{eV}$, respectively. Note that we are using model (C) for all the gain spectra presented in Fig.6 under the assumption that formation of $\mathrm{Nb}_{3} \mathrm{C}_{2}$ from fragmentation of higher mass clusters does not play a significant role in the formation of the gain spectra. From Fig.6 it can be clearly seen that the shape of the experimental gain spectra for $\mathrm{Nb}_{3} \mathrm{C}_{2}$ in panels (a) and (b) where fragmentation rate is likely very low due to the low IR pulse energy, is the same as those in panels (c) 
and (d), where fragmentation rate is likely much higher. The same similarity between gain spectra is observed for $\mathrm{Nb}_{6} \mathrm{C}_{5}$ cluster (panels (e-h)).

For $\mathrm{Nb}_{3} \mathrm{C}_{2}$ transition bands originating from two electronic states can be distinguished. It can be clearly seen that with increasing IR laser pulse energy both bands are broadening, while the intensity ratio of the two changes in favor of the the low-energy transition band. We interpret this as a gradual increase in population of the previously unpopulated electronic level, a result of the increasing internal energy of the cluster. It is interesting to note that the width and the intensity of the transition band do not change monotonously from 19 to $61 \mathrm{~mJ}$ IR pulse energy. This points to a limitation of the energy that can be pumped in the clusters during the IR heating (see subsection $\mathbf{C}$ below).

In comparison to $\mathrm{Nb}_{3} \mathrm{C}_{2}$, the transition bands for $\mathrm{Nb}_{6} \mathrm{C}_{5}$ are broader. From Eq. (7) and Ref. 35 it directly follows that, even with the same coupling parameter, a system that has a higher number of vibrational modes will have broader transition bands.

The relatively broad bands result in a slight overlap between the corresponding transition bands, already for the unexcited cluster. This eventually results in formation of one continuous band at a $61 \mathrm{~mJ}$ IR pulse energy, where the contribution from each separate transition band can be recognized only by existence of the inflection area in the range from $\sim 4.8$ to $\sim 4.9 \mathrm{eV}$. When the pulse energy of the IR radiation increases, increasing the population of the previously unoccupied high-lying electronic levels, the transition bands expand even more whereas the lowenergy ones are getting more and more intense.

\section{Temperature evolution}

One of the fit variables of model $(\mathrm{C})$ investigated here is the electronic temperature, which we can now depict as a function of the IR laser pulse energy. The results for $\mathrm{Nb}_{3} \mathrm{C}_{2}$ and $\mathrm{Nb}_{6} \mathrm{C}_{5}$ are shown in Fig. 7. For both clusters nonlinear behavior is observed. The curve for $\mathrm{Nb}_{3} \mathrm{C}_{2}$ appears to saturate when the pulse energy exceeds 19 mJ, with temperatures on the order of $1000 \mathrm{~K}$. Taking into account that clusters are ionized around $35 \mu$ s after IR excitation, we can assume that all redistribution processes are finished at the moment of probing and the electronic and vibrational temperatures are in equilibrium. Assuming this equilibrium, we calculated the total internal energies for both cluster sizes as the sum of the electronic and vibrational energies. For the vibrational system we follow the Dulong-Petit law and estimate the energy deposited into the cluster as $k_{B} T$ per vibrational degree of freedom. The energy contained in the electronic system is calculated as a sum of the product of energy and occupation probability of the individual levels lying above the HOMO. The total internal energies of $\mathrm{Nb}_{3} \mathrm{C}_{2}$ and $\mathrm{Nb}_{6} \mathrm{C}_{5}$ for various IR pulse energies are presented in Table I. The calculations yield the highest internal

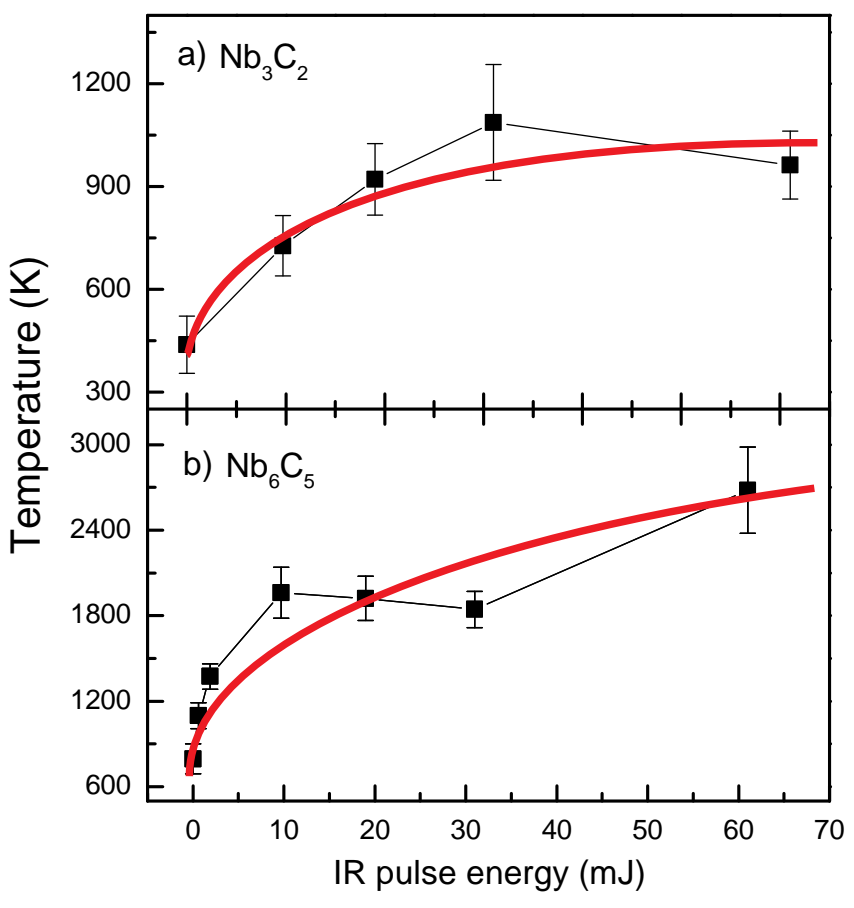

FIG. 7. Estimated electronic temperature of $\mathrm{Nb}_{6} \mathrm{C}_{5}$ (a) and $\mathrm{Nb}_{3} \mathrm{C}_{2}$ (b) clusters as a function of the IR pulse energy. Dots - values extracted from model (C), red line - guide to the eye.

energy of $0.9 \mathrm{eV}$ for $\mathrm{Nb}_{3} \mathrm{C}_{2}$; for $\mathrm{Nb}_{6} \mathrm{C}_{5}$, temperatures exceeding $2000 \mathrm{~K}$ are observed, corresponding to internal energies of $5-6 \mathrm{eV}$.

If higher internal energies than the ones observed here were produced by an increase of IR laser fluence, the clusters would exhibit fragmentation or direct ionization. But since the UV detection scheme is blind to direct ionization and/or fragmentation at timescales shorter than $10 \mu \mathrm{s}$, for which much higher internal energies are required, the limiting temperature can be interpreted as a maximum internal temperature for survival at $\mu \mathrm{s}$ timescales. The indication that fragmentation and/or ionization take place is found in the reduction of the number of detected clusters above the ionization threshold in Fig. 3 for $\mathrm{Nb}_{3} \mathrm{C}_{2}$.

Another way to estimate the maximum temperature for clusters to survive on the $35 \mu$ s timescale can be made using the theory of unimolecular decay ${ }^{38}$. Following this approach we obtained a temperature of approximately $2900 \mathrm{~K}$ for both cluster sizes for the direct ionization process. This value is on average higher than those reported in Table I. With this estimate, it appears reasonable that the clusters can withstand the temperatures found from model $\mathrm{C}$ on the timescale of the experiment without direct ionization. Since the temperatures for $\mathrm{Nb}_{3} \mathrm{C}_{2}$ are substantially lower, it seems that the loss observed for $\mathrm{Nb}_{3} \mathrm{C}_{2}$ is dominated by fragmentation. However, since the dissociation energies for $\mathrm{Nb}_{3} \mathrm{C}_{2}$ are as yet not reported, this remains speculative.

A better estimate for the internal energy of a cluster 


\begin{tabular}{lll} 
IR pulse energy $(\mathbf{m J})$ & $\mathbf{T}_{e}(\mathbf{K})$ & $\mathbf{E}_{\text {tot }}(\mathbf{e V})$ \\
\hline $\mathrm{Nb}_{3} \mathrm{C}_{2}$ & & \\
\hline $\mathrm{No} \mathrm{IR}$ & 438 & 0.34 \\
9.7 & 727 & 0.59 \\
19 & 921 & 0.77 \\
31 & 1087 & 0.93 \\
61 & 963 & 0.81 \\
\hline $\mathrm{Nb}_{6} \mathrm{C}_{5}$ & & \\
\hline $\mathrm{No} \mathrm{IR}$ & 796 & 1.89 \\
0.6 & 1099 & 2.65 \\
1.9 & 1374 & 3.36 \\
9.7 & 1961 & 4.92 \\
19 & 1922 & 4.81 \\
31 & 1844 & 4.60 \\
61 & 2680 & 6.88 \\
\hline
\end{tabular}

TABLE I. Values of the electronic temperature $\left(\mathrm{T}_{e}\right)$ extracted from model $\mathrm{C}$ and the corresponding total energy $\left(\mathrm{E}_{t o t}\right)$ for $\mathrm{Nb}_{3} \mathrm{C}_{2}$ and $\mathrm{Nb}_{6} \mathrm{C}_{5}$ for different IR pulse energies.

as a function of the IR pulse energy could be obtained by solving a set of coupled excitation rate equations ${ }^{39,40}$ which in our case can not be done due to the lack of information about absorption properties and the anharmonicity parameters for NbC clusters. Such a model is thus outside the scope of the current work.

\section{CONCLUSIONS}

We apply IR-UV spectroscopy for determination of the geometric structure of neutral $\mathrm{NbC}$ clusters and demonstrated the possibility to probe the excited electronic states close to the ionization threshold for $\mathrm{Nb}_{3} \mathrm{C}_{2}$ and $\mathrm{Nb}_{6} \mathrm{C}_{5}$ clusters. An explanation of the features on the UV-photoionization curves of a vibrationally pumped cluster has to incorporate both electronic and vibrational subsystems of the cluster. In accordance with this statement, it has been shown that the DFT-calculated eigen energies of Kohn-Sham orbitals in combination with the Fermi-Dirac distribution function can be used as a basis for construction of a transition band structure explaining every photoionization curve. The model has been shown to work on the example of two clusters: $\mathrm{Nb}_{3} \mathrm{C}_{2}$ and $\mathrm{Nb}_{6} \mathrm{C}_{5}$.

The population of the electronic states by manipulation of the vibrational coordinates observed for the clusters of NbC can be treated as a vibronic coupling and in principle can be investigated in a time resolved experiment. For this, one single sub-picosecond micropulse out of the FEL microsecond train has to be selected to achieve a proper time resolution. For $\mathrm{Nb}_{6} \mathrm{C}_{5}$ repopulation of the electronic state has been shown to take place even with an excitation energy of $0.6 \mathrm{~mJ}$ per pulse (Fig. 6(e)), which approximately corresponds to the energy of a single micropulse of the Free Electron Laser for Intra-Cavity Experiments (FELICE ${ }^{39}$ ).

\section{ACKNOWLEDGMENTS}

We gratefully acknowledge the Stichting voor Fundamenteel Onderzoek der Materie (FOM) for the support of the FELIX Laboratory. We thank the Netherlands Organization for Scientific Research Physical Sciences (NWOEW) and the SurfSara Supercomputer Center for providing the computational resources. We thank A. Diaz Bachs for assistance in the preparation of the experiments and E. Muskens for technical support.

${ }^{1}$ N. Ashcroft and N. Mermin, Solid State Physics (Saunders College, Philadelphia, 1976).

${ }^{2}$ W. Domcke, D. R. Yarkony, and H. Koppel, Conical intersections (World Scientific Publishing Co. Pte. Ltd., Singapore, 2011).

${ }^{3}$ E. Cappelluti and L. Pietronero, "Electron-phonon renormalization in small fermi energy systems," Phys. Rev. B 68, 224511 (2003).

${ }^{4}$ A. K. Geim and K. S. Novoselov, "The rise of graphene," Nat. Mat. 6, 198 (2007).

${ }^{5}$ S. Yang and M. B. Knickelbein, "Photoionization studies of transition metal clusters: Ionization potentials of $\mathrm{Sc}_{\mathrm{n}} \mathrm{O}(\mathrm{n}=5-36)$, , J. Chem. Phys. 93, 1533 (1990).

${ }^{6} \mathrm{~W}$. A. de Heer, "The physics of simple metal clusters: experimental aspects and simple models," Rev. Mod. Phys. 65, 611 (1993).

${ }^{7}$ H. Hohmann, C. Callegari, S. Furrer, D. Grosenick, E. E. B. Campbell, and I. V. Hertel, "Photoionization and fragmentation dynamics of $\mathrm{C}_{60}$," Phys. Rev. Lett. 73, 1919 (1994).

${ }^{8}$ E. E. B. Campbell, G. Ulmer, and I. V. Hertel, "Delayed ionization of $\mathrm{C}_{60}$ and $\mathrm{C}_{70}$," Phys. Rev. Lett. 67, 1986 (1991).

${ }^{9}$ K. Hansen, J. U. Andersen, P. Hvelplund, S. P. Møller, U. V. Pedersen, and V. V. Petrunin, "Observation of a 1/t decay law for hot clusters and molecules in a storage ring," Phys. Rev. Lett. 87, 123401 (2001).

${ }^{10}$ B. Baguenard, J. C. Pinare, C. Bordas, and M. Broyer, "Photoelectron imaging spectroscopy of small tungsten clusters: Direct observation of thermionic emission," Phys. Rev. A 63, 023204 (2001).

${ }^{11}$ E. E. B. Campbell and R. D. Levine, "Delayed ionization and fragmentation en route to thermionic emission: Statistics and dynamics," Annu. Rev. Phys. Chem. 51, 65 (2000).

12 J. U. Andersen, E. Bonderup, and K. Hansen, "Thermionic emission from clusters," J. Phys. B-Atomic Mol. Opt. Phys. 51, R1 (2002).

${ }^{13}$ M. Maier, G. Wrigge, M. A. Hoffmann, P. Didier, and B. v. Issendorff, "Observation of Electron Gas Cooling in Free Sodium Clusters," Phys. Rev. Lett. 96, 117405 (2006).

${ }^{14}$ D. van Heijnsbergen, G. von Helden, M. A. Duncan, A. J. A. van Roij, and G. Meijer, "Vibrational spectroscopy of gas-phase metal-carbide clusters and nanocrystals," Phys. Rev. Lett. 83, 4983 (1999).

${ }^{15}$ G. von Helden, I. Holleman, G. M. H. Knippels, A. F. G. van der Meer, and G. Meijer, "Infrared resonance enhanced multiphoton ionization of fullerenes," Phys. Rev. Lett. 79, 5234 (1997).

${ }^{16}$ J. Jalink, J. M. Bakker, T. Rasing, and A. Kirilyuk, "Channeling Vibrational Energy To Probe the Electronic Density of States in Metal Clusters," J. Phys. Chem. Lett. 6, 750-754 (2015).

${ }^{17}$ G. von Helden, I. Holleman, M. Putter, A. van Roij, and G. Meijer, "Infrared resonance enhanced multi-photon ionization spectroscopy of $\mathrm{C}_{84}$," Chem. Phys. Lett. 299, 171-176 (1999).

${ }^{18}$ A. Fielicke, J. T. Lyon, M. Haertelt, G. Meijer, P. Claes, J. de Haeck, and P. Lievens, "Vibrational spectroscopy of neutral silicon clusters via far-IR-VUV two color ionization," J. Chem. Phys. 131, 171105 (2009). 
${ }^{19}$ D. Oepts, A. F. G. van der Meer, and P. W. van Amersfoort, "The Free-Electron-Laser User Facility FELIX," Infrared Phys. \& Techn. 36, 297-308 (1995).

${ }^{20}$ G. Kresse and J. Furthmüller, "Efficient iterative schemes for ab initio total-energy calculations using a plane-wave basis set," Phys. Rev. B 54, 11169-11186 (1996).

${ }^{21}$ P. E. Blöchl, "Projector augmented-wave method," Phys. Rev. B 50, 17953-17979 (1994).

${ }^{22}$ G. Kresse, "From ultrasoft pseudopotentials to the projector augmented-wave method," Phys. Rev. B 59, 1758-1775 (1999).

${ }^{23}$ J. P. Perdew and Burke, "Generalized Gradient Approximation Made Simple," Phys. Rev. Lett. 77, 3865-3868 (1996).

${ }^{24}$ R. L. Johnston, "Evolving better nanoparticles: Genetic algorithms for optimising cluster geometries," Dalton Transactions , 4193 (2003).

${ }^{25}$ R. Logemann, G. A. de Wijs, M. I. Katsnelson, and A. Kirilyuk, "Geometric, electronic, and magnetic structure of $\mathrm{Fe}_{\mathrm{x}} \mathrm{O}_{\mathrm{y}}{ }^{+}$ clusters," Phys. Rev. B 92, 144427 (2015).

${ }^{26}$ N. Fukushima, K. Miyajima, and F. Mafuné, "Ionization energies of niobium carbide clusters $\mathrm{Nb}_{\mathrm{n}} \mathrm{C}_{\mathrm{m}}(\mathrm{n}=3-10, \mathrm{~m}=0-7)$," J. Phys. Chem. A 113, 2309-2315 (2009).

${ }^{27}$ D. van Heijnsbergen, A. Fielicke, G. Meijer, and G. von Helden, "Structure Determination of Gas-Phase Niobium and Tantalum Carbide Nanocrystals via Infrared Spectroscopy," Phys. Rev. Lett. 89, 013401 (2002).

${ }^{28}$ D.-S. Yang, M. Z. Zgierski, A. Berces, P. A. Hackett, P.-N Roy, A. Martinez, J. T. Carrington, D. R. Salahub, R. Fournier, T. Pang, and C. Chen, "Vibrational and geometric structures of $\mathrm{Nb}_{3} \mathrm{C}_{2}$ and $\mathrm{Nb}_{3} \mathrm{C}_{2}{ }^{+}$from pulsed field ionization-zero electron kinetic energy photoelectron spectra and density functional calculations," J. Chem. Phys. 105, 10663-10671 (1996).

${ }^{29}$ M. B. Knickelbein and S. Yang, "Photoionization and fragmentation dynamics of $\mathrm{C}_{60}$," J. Chem. Phys. 93, 5760-5767 (1990).
${ }^{30}$ R. Fowler, "Photoelectric sensitivity," Phys. Rev. 38, 107 (1931).

${ }^{31}$ A. Prem and V. V. Kresin, "Photoionization profiles of metal clusters and the Fowler formula," Phys. Rev. A 85, 8-11 (2012).

${ }^{32}$ M. Putter, G. von Helden, and G. Meijer, "Mass selective infrared spectroscopy using a free electron laser," 258, 118-122 (1996).

${ }^{33}$ J. Oomens, B. G. Sartakov, G. Meijer, and G. von Helden, "Gas-phase infrared multiple photon dissociation spectroscopy of mass-selected molecular ions," Int. J. Mass Spectrom. 254, 1-19 (2006).

${ }^{34}$ S. E. Stein and B. S. Rabinovitch, "Accurate evaluation of internal energy level sums and densities including anharmonic oscillators and hindered rotors," J. Chem. Phys. 58, 2438 (1973).

${ }^{35}$ H. G. Limberger and T. P. Martin, "Ionization energies of cesium and cesium oxide clusters," J. Chem. Phys. 90, 2979 (1989).

${ }^{36}$ T. Bergmann and T. P. Martin, "Observation of electronic shell structure in large cesium oxide clusters," J. Chem. Phys. 90, 2848 (1989).

${ }^{37}$ V. Dryza, M. A. Addicoat, J. R. Gascooke, M. A. Buntine, and G. F. Metha, "Threshold photoionization and density functional theory studies of the niobium carbide clusters $\mathrm{Nb}_{3} \mathrm{C}_{n}(\mathrm{n}=1$ 4) and $\mathrm{Nb}_{4} \mathrm{C}_{n},(\mathrm{n}=1-6), "$ J. Phys. Chem. A 112, 5582-5592 (2008).

${ }^{38}$ K. Hansen, Statistical Physics of Nanoparticles in the Gas Phase (Springer, The Netherlands, 2013).

39 J. M. Bakker, V. J. F. Lapoutre, B. Redlich, J. Oomens, B. G. Sartakov, A. Fielicke, G. von Helden, G. Meijer, and A. F. G. van der Meer, "Intensity-resolved IR multiple photon ionization and fragmentation of $\mathrm{C}_{60}$," J. Chem. Phys. 132, 1-9 (2010).

${ }^{40}$ F. Calvo, Y. Li, D. M. Kiawi, J. M. Bakker, P. Parneix, and E. Janssens, "Nonlinear effects in infrared action spectroscopy: Experiment and kinetic modeling," Phys. Chem. Chem. Phys. 17, 25956-25967 (2015) 